\title{
Non-Hodgkin's Lymphoma Masquerading as Psoas Abscess: A Case Report
}

\author{
WM Ng, MS Ortho (UM), TC Ong*, MRCP, MK Kwan, MS Ortho (UM), CY Cheok**, MS Ortho (UM) \\ Department of Orthopaedic Surgery, University Malaya Medical Centre, Kuala Lumpur, Malaysia \\ * Haematology unit, Medical Department, Hospital Kuala Lumpur, Malaysia \\ ** Department of Orthopaedics, University Putra Malaysia, Hospital Kuala Lumpur, Malaysia
}

\begin{abstract}
We report here a rare presentation of an extra-nodal nonHodgkin's lymphoma. Both clinical presentation radiological findings were suggestive of psoas abscess. Surgical debridement was performed and histopathological examination of the tissue sample revealed the diagnosis of non-Hodgkin's lymphoma. It is therefore important to note that non-Hodgkin's lymphoma can mimic psoas abscess and that psoas lesion with vertebral involvement does not necessarily indicate infection. It is always advisable to obtain needle biopsy to establish diagnosis before embarking on surgical intervention. The practice to send any suspicious tissue obtained intra-operatively for histopathological examination is also warranted.
\end{abstract}

Key Words:

Non Hodgkin's Lymphoma, Psoas Abscess

\section{CASE REPORT}

Mr X, a 29 y old general worker presented with 2 months history of low backache associated with left thigh pain. He also reported persistent low-grade fever associated and significant weight loss (more than $5 \mathrm{~kg}$ ). The left thigh pain progressively worsened in the two weeks prior to presenting at the clinic and he started to experience difficulty in walking; however there was no history of lower limb numbness or weakness. The patient reported no history of chronic cough, shortness of breath or haemoptysis. He had neither tuberculosis infection nor contact with tuberculosis patients.

Clinically he looked pale and abdominal examination revealed a tender palpable mass felt over the left iliac fossa region that extended into the pelvic region. The left hip was stiff and was kept in flexed and externally rotated position. The psoas sign was positive. Spinal examination revealed tenderness over the thoracolumbar junction. Curvature of the spine was within normal limits but range of motion was limited. Neurological examination of both lower limbs was normal. There were however multiple small palpable lymph nodes in both inguinal regions and axilla. Chest examination revealed no abnormality.

Total white cell count was high at $19.8 \times 10^{9} / 1$. The patient was anaemic with a haemoglobin level of $9.3 \mathrm{~g} / \mathrm{dl}$ and platelets were high at $638 \times 10^{\circ} / 1$. The C-reactive protein (CRP) level was $6.97 \mathrm{mg} / \mathrm{dl}$ with a very high Erythrocyte Sedimentation Rate (ESR) of $120 \mathrm{~mm} / \mathrm{hr}$. The Mantoux test was negative. Blood culture and sensitivity test was also negative. The peripheral blood picture showed normocyticnormochromic anaemia. There was leucocytosis with the presence of the toxic looking neutrophils and reactive thrombocytosis indicative of an infection or and/or inflammation. The radiograph of the thoracolumbar spine (Fig. 1) revealed erosion of the anterior vertebral body of the L1 and L2 vertebra with sparing of the end plate of the both vertebra. There was no abnormal lesion seen in the respective vertebra and vertebral heights were normal. Computerized tomography (CT) scan of the thoracolumbar spine (Fig.2) revealed an inflammatory collection within the left psoas muscle and confirmed erosion of anterior portion of the L1 and $\mathrm{L} 2$ vertebra.

A provisional diagnosis of pyogenic spinal infection with left psoas abscess was offered. The patient was started on the empirical antibiotic therapy consisting of a combination of intravenous cloxacillin and gentamicin, however the pain and fever persisted despite antibiotic therapy. Surgical debridement and drainage of the left psoas abscess was performed 10 days after starting antibiotics. Intraoperatively, there was a $10 \times 5 \mathrm{~cm}$ lesion in the left psoas muscle filled with unhealthy reddish looking inflammatory tissue, some necrotic tissue and purulent discharge. The tissue was sent for culture and histopathological examination.

Postoperatively, the patient was able to stand upright with near normal hip motion. The tissue culture report showed no growth detected. Histopathological examination revealed a high grade T-cell, anaplastic large cell lymphoma, with 


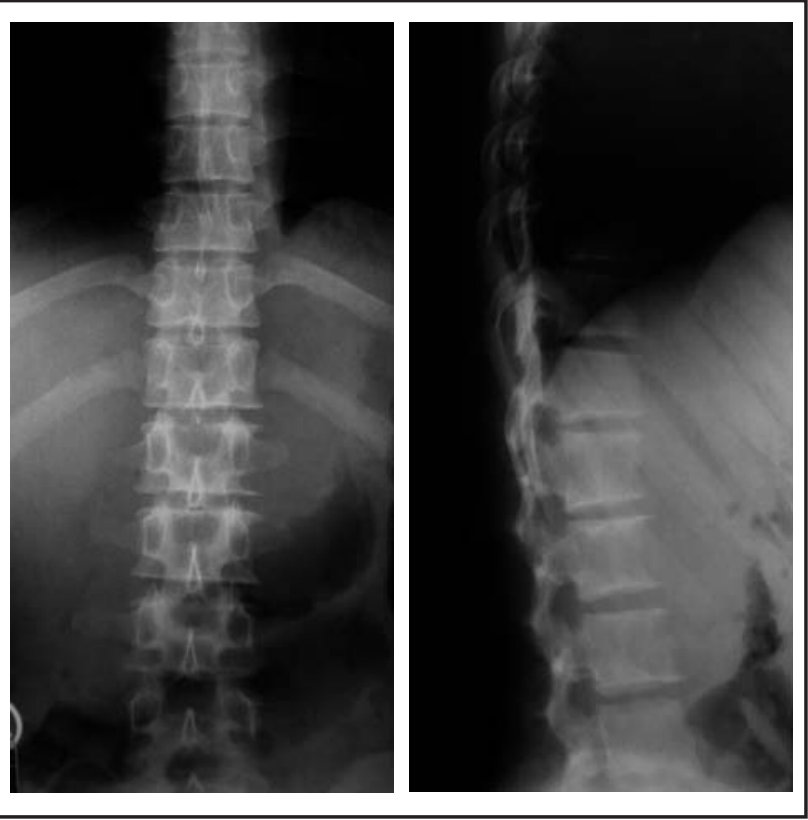

Fig. 1: Anteroposterior view of the thoracolumbar radiographs showed the obliteration of the shadow of left psoas muscle and lateral view demonstrates bony erosion over the anterior border of the L1 and L2 vertebra.

positive immunohistochemical staining for CD 30, EMA and ALK1. He was then referred to the haematology team for further evaluation and management. The patient's symptoms and general condition improved tremendously after a few cycles of chemotherapy, consists of cyclophosphamide, adriamycin, oncovin and prednisoslone (CHOP). He was then scheduled for bone marrow transplant with the goal of achieving long-term remission.

\section{DISCUSSION}

Approximately $25 \%$ of non-Hodgkin's lymphoma cases present extranodally ${ }^{1}$; in fact, liver, spleen, gastrointestinal organs and kidney are common sites of involvement. Primary lymphoma involving the musculoskeletal system is rare. The primary sites for bone lymphoma are ileum, scapula, vertebrae, femur or tibia whereas the thigh, calf and the upper arm muscles are the usual sites for skeletal muscle lymphoma. Primary involvement of the psoas muscle as in the case presented herein is quite rare. Glazer et al reported $4 \%$ of non-Hodgkin's lymphoma patients undergoing abdominal CT staging had secondary psoas muscle involvement ${ }^{2}$, but primary lymphoma of the psoas muscle is indeed very rare.

Clinical symptoms and signs of this case were indeed diagnostic of the psoas abscess following a spinal infection. Tumour was not considered as a diagnosis because spinal infection especially tubercular infection is quite common in this part of world. Moreover, primary lymphoma involving a psoas muscle is quite an atypical presentation.

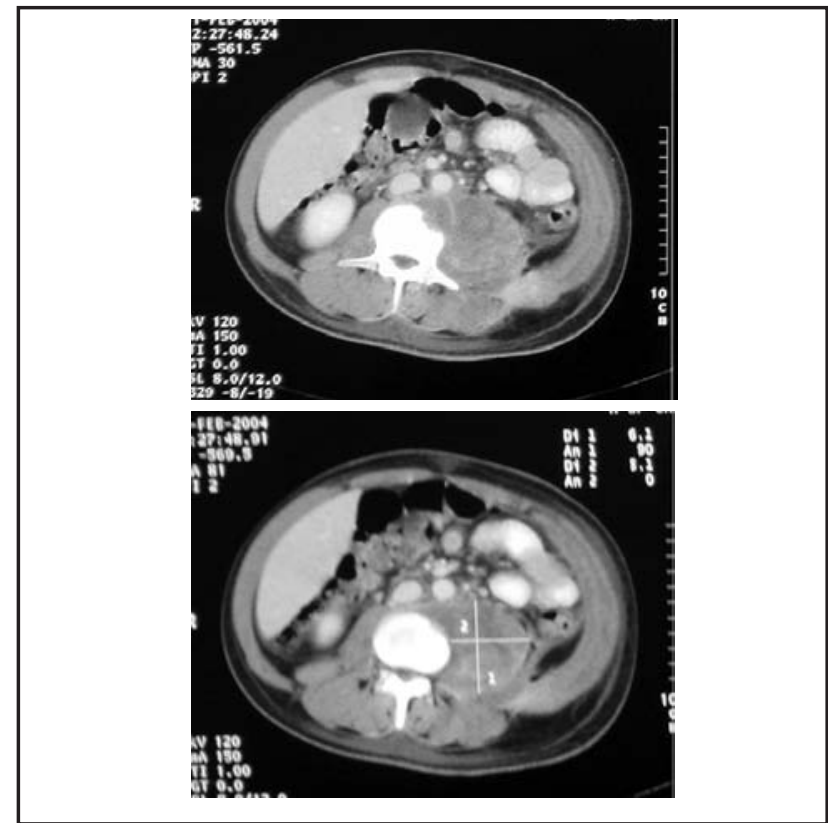

Fig. 2: CT scan at the level of L1 revealed bony erosion of anterior aspect of the L1 vertebral body and the left psoas lesion.

Retrospectively, the presence of bilateral inguinal and axilla lymphadenopathy should have alerted us to the possibility of centripetal distribution of Non-Hodgkin's lymphoma. The fever (Pel-Ebstein fever) and weight loss were indeed systemic symptoms of the lymphoma. Although the blood count, C-reactive protein (CRP), Erythrocyte Sedimentation Rate (ESR) and peripheral blood picture corresponded with an infectious causation, the absence of positive blood culture should have reminded us to review the diagnosis. Radiographs and CT scan findings did not reveal classical destruction at the site of the suspected spinal infection (usually involving the end plate and/ or disc space). CT guided needle biopsy should be performed prior to surgical management. Failure of empirical antibiotic treatment further indicated that the infection might not have been the correct diagnosis. During surgical intervention, the presence of unhealthy reddish looking inflammatory tissue with the absence of purulence should suggest to the surgeon that he is dealing with a non-infectious condition. It is always advisable to send tissue for culture and sensitivity as well as histopathological examination in all psoas abscess cases.

This case illustrates the diagnostic difficulty associated with a rare presentation of non-Hodgkin's lymphoma of the psoas muscle that closely mimics psoas abscess. A psoas lesion with vertebral involvement does not necessarily mean infection. Therefore, CT guided needle biopsy is indicated for this situation and surgical intervention may have been avoided. The practice of sending suspicious tissue obtained intra-operatively for histopathological examination should be a standard practice. 


\section{REFERENCES}

1. Freeman C, Berg JW, Cutler SJ. Occurrence and prognosis of extra-nodal lymphomas. Cancer. 1972; 29: 252-60.

2. Glazer HS, Lee JKT, Balfe DM. Non Hodgkin's lymphoma: computed tomographic demonstration of the unusual extra-nodal involvement. Radiology. 1983; 149: 211-7.

3. Hopkins TJ, Raducan V, Sioutos N, White A. Lumbar lymphoma as psoas abscess/epidural mass with acute cauda equina syndrome. A case report. Spine. 1993 May; 18(6): 774-8. 